\title{
Molecular dynamics simulation of hydrogen and helium trapping in tungsten
}

\author{
Petr Grigoreva,b,c,*, Aleksandr Zinoveve, ${ }^{\mathrm{a}, \mathrm{d}, \mathrm{e}}$, Dmitry Terentyev ${ }^{\mathrm{a}}$, Giovanni \\ Bonny $^{\mathrm{a}}$, Evgeny E. Zhurkin ${ }^{\mathrm{c}}$, Guido Van Oost ${ }^{\mathrm{b}}$, Jean-Marie Noterdaeme ${ }^{\mathrm{f}}$ \\ ${ }^{a} S C K \bullet C E N$, Nuclear Materials Science Institute, Boeretang 200, Mol, 2400 Belgium \\ ${ }^{b}$ Ghent University, Applied Physics EA17 FUSION-DC, St.Pietersnieuwstraat 41, Gent, B4 \\ B-9000 Belgium \\ ${ }^{c}$ Department of Experimental Nuclear Physics K-89, Institute of Physics, Nanotechnologies \\ and Telecommunications, Peter the Great St.Petersburg Polytechnic University, St. \\ Petersburg, Russia \\ ${ }^{d}$ Université catholique de Louvain, Place de l'Université 1, B-1348 Louvain-la-Neuve, \\ Belgium \\ ${ }^{e}$ Institute for Metals Superplasticity Problems, Russian Academy of Sciences, ul. S. \\ Khalturina 39, Ufa, 450001 Russia \\ ${ }^{f}$ Max-Planck-Institut für Plasmaphysik, Garching, Germany
}

\begin{abstract}
Tungsten has been chosen as the divertor armour material in ITER and is the main candidate material for plasma-facing components for future fusion reactors. Interaction of plasma components with the material leads to degradation of the performance and thus the lifetime of the in-vessel components. On top of that special attention is drawn to tritium retention in the reactors vessel from a safety point of view, since tritium is radioactive material. In order to gain better understanding of the mechanisms driving accumulation of plasma components in the material and subsequent degradation of the material, atomistic simulations are employed. The focus of this work is on so-called self trapping of $\mathrm{H}$ and $\mathrm{He}$ atoms or, in other words, Frenkel pair formation in bulk tungsten in the presence of $\mathrm{H}$ and $\mathrm{He}$ atoms. Two versions of a model embedded atom interatomic potential and a bond order potential were tested by comparing it with $a b$ initio data regarding the binding properties of pure He and He-H-Vacancy clusters and energetics of Frenkel pair formation. As a result of Molecular Dynamics simulations at finite temperature, the values of critical $\mathrm{H}$ concentration
\end{abstract}

*Corresponding author 
needed for the generation of a Frenkel pair in the presence of He clusters were obtained. The results show that the critical $\mathrm{H}$ concentration decreases with the size of He cluster present in the simulation cell and thus, Frenkel pair formation by $\mathrm{H}$ is facilitated in the presence of He clusters in the material.

Keywords: tungsten, plasma facing material, hydrogen retention, helium, molecular dynamics

\section{Introduction}

Plasma-facing materials in a fusion device are exposed to extreme conditions in terms of heat and particle loads as well as to high energy neutron irradiation. As a consequence of the fact that plasma-facing materials are in direct contact

5 with plasma, a failure of the material can lead to a catastrophic event for the device. Due to its outstanding thermal properties, tungsten (W) was chosen as the divertor armour material for ITER - the next generation tokamak that is currently under construction, and DEMO - future demonstration nuclear fusion power station [1].

Together with demonstration and testing the technologies for DEMO, one of the main goals of operation of ITER is to demonstrate the control of the fusion plasma with negligible consequences for the environment. From this point of view special attention is drawn to tritium $(\mathrm{T})$, which is a radioactive hydrogen $(\mathrm{H})$ isotope and is a part of the fusion fuel. Consequently, the retention of ${ }_{15} \mathrm{~T}$ in the plasma-facing material is a safety issue, hence a limit of $700 \mathrm{~g}$ of $\mathrm{T}$ accumulated in the reactor chamber was set for ITER by the safety authorities 2. Helium (He) is produced in fusion reactions inside the plasma and also in transmutation reactions of $\mathrm{W}$ caused by high-energy neutrons being a product of the fusion reaction. Thus, both $\mathrm{T}$ and $\mathrm{He}$ will be present in the material 20 during the device operation. Therefore it is important to investigate possible synergistic effects between $\mathrm{T}$ and He both in terms of $\mathrm{T}$ retention and possible detrimental effects on the properties of the plasma facing material.

The effect of $\mathrm{H}$ plasma exposure on $\mathrm{W}$ has been extensively studied in experi- 
ments involving deuterium plasma 3 11. H concentration can easily exceed the solubility limit in the subsurface area during high flux plasma exposure. This leads to $\mathrm{H}$ bubble and void formation occurring at a depth of several micrometers, which exceeds the implantation depth of a few nanometers. Experiments involving $\mathrm{He}$ implantation demonstrate the presence of $\mathrm{He}$ bubbles and 'fuzz' formation at a length scale comparable to the implantation depth [12 14].

However systematic studies are scarce and large scatter in the data is usually attributed to different material preparation procedures, material microstructure and impurity levels in different experiments [8]. Experimental studies involving simultaneous $\mathrm{H}$ and He plasma exposure [15, 16] demonstrated suppressed blister formation as compared to pure $\mathrm{H}$ plasma exposure. This effect was at35 tributed to a decrease of $\mathrm{H}$ permeability through the subsurface region due to He bubble formation. Another remarkable effect was the detection of nanometric He bubbles at a depth significantly larger than the He implantation range [15], not seen in pure He exposures.

$\mathrm{H}$ and $\mathrm{He}$ behaviour in $\mathrm{W}$ has been also investigated by means of atomistic simulations, a thorough review of recent modelling activities can be found in [17] and [18, 19. According to ab initio studies both $\mathrm{H}$ and He atoms occupy tetrahedral interstitial position in bulk $\mathrm{W}$ with a very low migration barrier between the positions [20 23]. At the same time clustering behaviour of $\mathrm{H}$ or $\mathrm{He}$ atoms in bulk $\mathrm{W}$ is essentially different: $\mathrm{H}$ atoms show practically zero interac-

45 tion while He atoms exhibit strong binding leading to so-called self-trapping He bubble formation mechanism [24]. Ab initio studies [24] 26] showed that there is an attractive interaction between He clusters and $\mathrm{H}$ atoms in bulk W. However, comprehensive physical mechanisms leading to synergistic effects during simultaneous $\mathrm{H}$ and He plasma exposure at elevated temperature are not clear 50 so far.

In the present work, the $\mathrm{H}$ bubble nucleation in $\mathrm{W}$ in the presence of $\mathrm{He}_{\mathrm{M}}(\mathrm{M}$ - number of atoms in the cluster) clusters was assessed by means of Molecular Statics (MS) and Molecular Dynamics (MD) simulations. The impact of the $\mathrm{H}$ implantation rate and the size of $\mathrm{He}_{\mathrm{M}}$ clusters $(\mathrm{M})$ present in the system on $\mathrm{H}$ 
bubble nucleation was investigated. The obtained MS results were compared with $a b$ initio data in order to validate the choice of the interatomic potential. MD simulations allowed us to detect and investigate the conditions for $\mathrm{H}$ bubble nucleation in terms of critical $\mathrm{H}$ concentration depending on the content of $\mathrm{He}$ in the system. This study gives an insight about mechanisms of $\mathrm{H}$ bubble

${ }_{60}$ formation during mixed He-H plasma exposure and, together with the data for diffusion and thermal stability of mixed $\mathrm{He}_{\mathrm{M}}-\mathrm{H}_{\mathrm{N}}$ clusters reporter earlier [27], provides the parameters needed for larger length and longer time scale models required for realistic plasma exposure simulations.

\section{Computational details}

The Molecular Static calculations were performed using interatomic potentials for the W-H-He system developed in the frameworks of two different models: Bond Order Potentials (BOP) by Li et al. [28, 29] and Embedded Atom Method (EAM) developed by Bonny et al. [30]. The BOP potential was fitted with special attention on reproducing defect formation energies and interaction of $\mathrm{H}$ and $\mathrm{He}$ with the point defects in W. Both EAM potentials were created aiming at the investigation of the interaction of $\mathrm{H}$ and $\mathrm{He}$ with dislocations in tungsten and are based on the interatomic potential for $\mathrm{W}-\mathrm{W}$ interaction named "EAM2" developed by Marinica et al. [31. The base W-W potential was selected after critical review of 19 different EAM potentials with special attention to properties of screw dislocations given in 32 . There are two different versions of the EAM potential, referred to as "EAM1" and "EAM2" in 30.

For the EAM1 potential, emphasis was put on a quantitative reproduction of ab initio data for the binding between $\mathrm{H}-\mathrm{H}, \mathrm{He}-\mathrm{He}$ and $\mathrm{He}-\mathrm{H}$ pairs in the bulk tungsten. The off-centre position of a $\mathrm{H}$ atom in a vacancy as predicted by Density Functional Theory (DFT) [21] was not considered, and therefore both $\mathrm{H}$ and $\mathrm{He}$ are described by pair potentials only.

For EAM2 the focus was made on stabilising a $\mathrm{H}$ atom in an off-centre position in the vacancy and therefore an embedding function was added for 
H. All types of the potentials predict the tetrahedral position for a $\mathrm{H}$ atom as the most favourable in bulk W. The main goal of the MS calculations was to compare and validate the interatomic potentials using ab initio data available in literature and choose the potential for further Molecular Dynamic simulations at finite temperature.

The size of the crystallite used in the simulations was $10 \times 10 \times 10 a_{0}^{3}\left(a_{0}\right.$ is the lattice constant predicted by the potential: 3.14 and $3.165 \AA$ for EAM and BOP potentials respectively). It contained 2000 atoms before any point defect or cluster was introduced. Periodic boundary conditions were applied in all three directions. The incremental binding energy of $\mathrm{H}(\mathrm{He})$ atom to a $\mathrm{He}_{\mathrm{M}}-\mathrm{Vac}$ cluster is defined as an energy difference between the system where a $\mathrm{H}(\mathrm{He})$ 95 atom is part of the cluster $\mathrm{H}(\mathrm{He})-\mathrm{He}_{\mathrm{M}^{-}} \mathrm{Vac}$ and a system where the $\mathrm{H}(\mathrm{He})$ atom is far away from the cluster in equilibrium tetrahedral position and can be written as:

$$
E_{b}=E_{\text {tetra }}+E_{\mathrm{He}_{\mathrm{M}}-\mathrm{Vac}}-E_{\mathrm{H}(\mathrm{He})-\mathrm{He}_{\mathrm{M}}-\mathrm{Vac}}-E_{r e f}
$$

where $E_{\mathrm{H}(\mathrm{He})-\mathrm{He} \text {-Vac }}$ is the total energy of the system containing $\mathrm{H}(\mathrm{He})$ $\mathrm{He}_{\mathrm{M}}$-Vac cluster, $E_{\text {tetra }}$ - energy of the system with $\mathrm{H}(\mathrm{He})$ atom in a tetrahedral interstitial position, $E_{\mathrm{He}_{\mathrm{M}} \text {-Vac }}$ is the energy of the system containing a $\mathrm{He}_{\mathrm{M}}-\mathrm{Vac}$ cluster and $E_{r e f}$ is the energy of defect free W lattice without any $\mathrm{H}$ or $\mathrm{He}$ atoms and is added to complete the energy balance. In this notation a positive value indicates attraction and a negative value indicates repulsion.

The so-called process of self-trapping and defect formation during plasma implantation can be seen as a $\mathrm{He}_{\mathrm{M}}$ cluster formation, and a consequent generation of a Frenkel pair (vacancy and self interstitial pair) assisted by the cluster. The Frenkel pair formation energy $E_{f}$ was studied by means of Molecular Statics calculations and was estimated via calculating the following energy balance:

$$
E_{f}=E_{\mathrm{He}_{\mathrm{M}}-\mathrm{Vac}}+E_{\mathrm{SIA}}-E_{\mathrm{He}_{\mathrm{M}}}-E_{r e f}
$$

where $E_{\mathrm{He}_{\mathrm{M}} \text {-Vac }}$ is the total energy of the box containing the $\mathrm{He}_{\mathrm{M}}$ cluster 
in a vacancy, $E_{\mathrm{He}_{\mathrm{M}}}$ and $E_{\mathrm{SIA}}$ is total energy of the system containing a $\mathrm{He}_{\mathrm{M}}$ cluster and a Self Interstitial Atom (SIA) in the form of a $\langle 111\rangle$ crowdion in bulk tungsten respectively, $E_{r e f}$ is the total energy of the box containing no defects (BCC W in our case). In this notation, a positive value of the formation energy corresponds to an endoergic process, i. e. a certain activation energy is necessary to create a Frenkel pair. Negative value of the formation energy corresponds to an exoergic process, i. e. energy is released during the reaction. The static relaxation was performed using the conjugate gradient algorithm embedded in LAMMPS simulation package 33. with a stopping criterion on the relative energy change of $10^{-10}$ between minimisation steps. For system containing 2000 atoms it corresponds to the error in total energy of $10^{-6} \mathrm{eV}$, however for larger systems the error will be larger. Prior to the static relaxation of the considered atomic configuration a short MD run at $300 \mathrm{~K}$ for 1 ps was performed after which the system was quenched to $0 \mathrm{~K}$. This procedure allows the possibility for the system to evolve out of local minima and arrange itself into the most stable configuration.

The computational cell for MD simulations was a cube $10 \times 10 \times 10 a_{0}^{3}$ in which the crystal's $\langle 100\rangle$ directions were aligned with the coordinate axes. Periodic boundary conditions were applied in all three directions. The needed number of He atoms (M) were assigned random positions in the crystal from the very beginning, and $\mathrm{H}$ atoms were added in the computation cell in random positions with periodic intervals of time. The temperature was kept constant equal to 800 $\mathrm{K}$ in all the simulations. The value of the temperature was chosen to ensure high mobility of the clusters during the simulations and corresponds to surface temperature range at divertor vertical targets expected for ITER baseline heat load scenario as predicted in 34]. The interatomic potential "EAM2" and NVT ensemble with Nosé-Hoover thermostat were used.

Two series of simulations were conducted: the first one was directed to study the effect of hydrogen insertion rate (HIR) on the ejection process, i. e. displacement of $\mathrm{W}$ atom from its equilibrium site, resulting in the formation of a self-interstital atom and a vacancy pair. In this case no He atoms were present 
and the a hydrogen atom was inserted in the system with a certain period that was varied from 10 to 90 ps that corresponds to HIR of 50 and 5.6 appm/ps respectively. The calculations were made in two types of simulation boxes: a perfect $\mathrm{W}$ box without defects and a $\mathrm{W}$ box containing one pre-existing vacancy in order to promote the ejection process. In the second series the effect of the initial number of $\mathrm{He}$ atoms in the system on the time it takes to nucleate the first bubble was investigated in the defect-free lattice. The initial number of $\mathrm{He}$ atoms varied from zero to six, the HIR was equal to $16.7,10$ or $5.6 \mathrm{appm} / \mathrm{ps}$. The He atoms were put at random positions in the beginning of the simulations. Given the size of the simulation box representing the material in a subsurface depth region the corresponding hydrogen flux is $\approx 10^{27} \frac{1}{m^{2} s}$ which is three orders of magnitude higher than the expected flux in ITER and accessible by the experiments. Such high values are typical for MD [35] and simulations with significantly lower values of the flux are prohibitively time consuming due to the limitations of computational resources.

In order to detect an atom ejection (Frenkel pair formation), the positions of the tungsten atoms in the simulation box were periodically compared with the corresponding ideal lattice during the post-processing stage. A lattice site was considered empty if a sphere of radius equal to $0.2 a_{0}$, encircled around the site, did not contain any tungsten atoms. The sphere radius was chosen to be slightly larger than the typical thermal oscillation amplitude, measured in a separate MD simulation. If the sphere remained empty during $80 \%$ of a 300 ps timespan, the corresponding tungsten atom was assumed to have been ejected and the lattice site was considered as "vacant", to filter out occasional leaps of tungsten atoms from their positions due to thermal vibration. The atomic concentration of $\mathrm{H}$ in the simulation box in that moment serves as a measure of the ease of atom ejection and is called "critical hydrogen concentration" in the present text. 


\section{Results and discussion}

170

3.1. Molecular Statics results

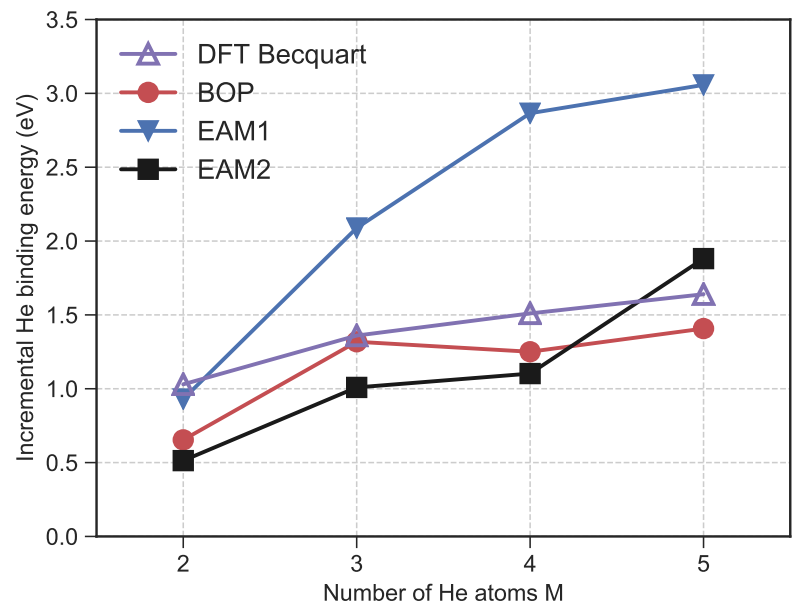

Figure 1: Incremental binding energy of a He atom to a $\mathrm{He}_{\mathrm{M}}$ cluster in perfect bulk tungsten. The hollow triangles represent DFT data from [24] and solid symbols show the results for three interatomic potentials: circles for BOP and triangles and squares for EAM1 and EAM2 respectively.

A set of MS calculations was done to test the performance of the interatomic potentials before launching computationally demanding dynamic simulations. Three main processes that define the trapping during mixed He-H implantation are: stability of $\mathrm{He}_{\mathrm{M}}$ clusters, stability of mixed $\mathrm{He}_{\mathrm{M}}-\mathrm{H}_{\mathrm{N}}$ clusters and Frenkel pair formation in the presence of $\mathrm{He}_{\mathrm{M}}$ clusters. The performance of the potentials regarding these three processes was assessed by calculating the incremental binding energy of a pure $\mathrm{He}_{\mathrm{M}}$ cluster, binding energy of a $\mathrm{H}$ atom to $\mathrm{He}_{\mathrm{M}}-\mathrm{H}-\mathrm{Vac}$ clusters and Frenkel pair formation energy and comparison of the results with $a b$ initio data from the literature.

The results of the potentials for the binding energy of a He atom to a $\mathrm{He}_{\mathrm{M}}$ cluster as a measure of the stability of $\mathrm{He}_{\mathrm{M}}$ clusters in bulk tungsten are shown in figure 1. It can be seen that EAM1 potential shows rapid increase of He 
binding energy with increasing number of $\mathrm{He}$ atoms $\mathrm{M}$ in the cluster $\mathrm{He}_{\mathrm{M}}$ and significantly overestimates the value of the binding energy as compared to $a b$ initio results. At the same time EAM2 and BOP potentials show good agreement with ab initio from [24, however with slight underestimation of the binding energy.

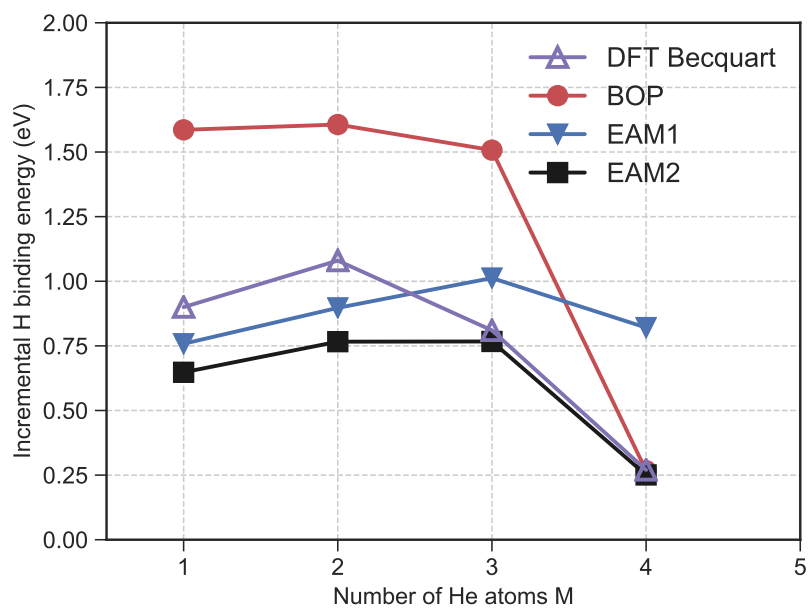

Figure 2: Binding energy of a $\mathrm{H}$ atom to a $\mathrm{He}_{\mathrm{M}}$-Vac cluster. The hollow triangles represent DFT data from [24] and solid symbols show the results for three interatomic potentials: circles for BOP and triangles and squares for EAM1 and EAM2 respectively.

The results for incremental binding energy of a $\mathrm{H}$ atom to a $\mathrm{He}_{\mathrm{M}^{-}}$Vac cluster are shown in figure 2. As can be seen from the figure, the BOP potential overestimates the binding energy almost by a factor of two for clusters containing one to three He atoms. This behaviour of the BOP potential is expected due to the overestimation of the $\mathrm{H}$ binding energy to a $\mathrm{H}_{\mathrm{N}}$-Vac cluster [36]. EAM1 gives reasonable agreement for clusters with one and two He atoms with an overestimation of the binding energy for bigger clusters, failing to reproduce the $a b$ initio trend of the binding energy drop for cluster with four atoms. At the same time the EAM2 potential reproduces the $a b$ initio trend well with some underestimations for small clusters. 
MS assessment of $\mathrm{He}_{\mathrm{M}}$ cluster assisted Frenkel pair formation was made by calculating the energy balance of a system containing a $\mathrm{He}_{\mathrm{M}}$ cluster in an ideal

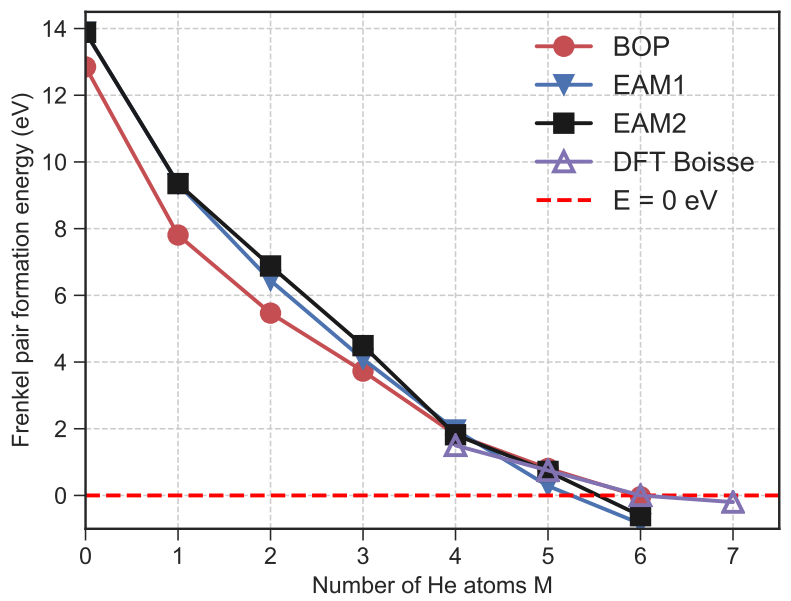

Figure 3: $\mathrm{He}_{\mathrm{M}}$ cluster assisted Frenkel pair formation energy. The hollow triangles represent DFT data from [37] and solid symbols show the results for three interatomic potentials: circles for BOP and triangles and squares for EAM1 and EAM2 respectively.

The potentials predict a value for $\mathrm{M}$ between five and six atoms as the threshold size of the $\mathrm{He}_{\mathrm{M}}$ cluster at which the formation of a Frenkel pair becomes energetically favourable. Despite the significant difference in description of bonding of $\mathrm{He}_{\mathrm{M}}$ clusters in bulk tungsten and $\mathrm{He}_{\mathrm{M}}-\mathrm{H}-\mathrm{Vac}$ clusters (see Figure 3), both versions of the EAM potentials together with BOP potential give very similar values for the formation energy of Frenkel pairs. Since there is no difference between both EAM and BOP potentials with respect to the He self- 
trapping mechanism, but the EAM2 potential describes the energetics of $\mathrm{He}_{\mathrm{M}}$ and $\mathrm{He}_{\mathrm{M}}-\mathrm{H}-\mathrm{Vac}$, the EAM2 potential was chosen for the finite temperature simulations.

\subsection{Molecular Dynamics results}

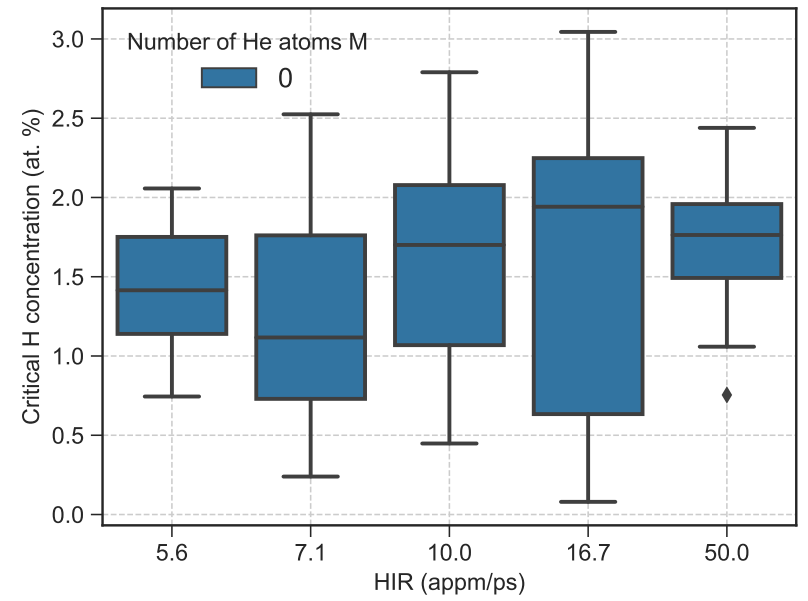

Figure 4: Critical average concentration of $\mathrm{H}$ in the absence of He atoms, but in the presence of a pre-existing vacancy. The data is presented using box-and-whiskers plot: the bottom and the top of the box denote the first and the third quartiles of the data correspondingly and thus indicate $\mathrm{H}$-spread or Interquartile Range (IQR), i.e. the range containing $50 \%$ of the values, the line inside the box represents the data median while whiskers span the rest of the dataset, the diamonds show outliers, i.e. the data points that lie further than $1.5 \times \mathrm{IQR}$ from either the first or the third quartile.

The results of the first series of MD simulations (without He atoms in a $\mathrm{W}$ box containing one pre-existing vacancy) revealing the effect of hydrogen insertion rate (HIR) on hydrogen critical concentration for Frenkel pair formation are shown in figure 4. The value of HIR was varied in the range between 5.6 and $50 \mathrm{appm} / \mathrm{ps}$ and each simulation was repeated 10 times with different values of the random generator seed used to generate initial atom velocity distributions to estimate the uncertainty of the results. Due to the small number of the data 
points, the best way to represent such dataset is so-called box-and-whiskers plot 38. The box-and-whiskers plot gives an opportunity to visually represent the data distribution properties such as spread and symmetry. The bottom and the top of the box denote the first and the third quartiles correspondingly and thus indicate $\mathrm{H}$-spread or Interquartile Range (IQR), i.e. the range containing $50 \%$ of the values. The line inside the box represents the data median while whiskers span the rest of the dataset. The data points, if present in the graph, show outliers, i.e. the data points that lie further than $1.5 \times \mathrm{IQR}$ from either the first or the third quartile. As can be seen from the figure, it is not possible to draw any trend in the data as the value of HIR increases, especially taking into account the IQR. Thus it was concluded that the variation of the HIR in the considered range does not affect the value of critical $\mathrm{H}$ concentration for Frenkel pair formation at a pre-existing vacancy in the case of simulations with $\mathrm{H}$ only.

As was discussed in the introduction, $\mathrm{H}$ and $\mathrm{He}$ atoms show significantly different clustering behaviour in bulk tungsten with a strong attraction between He atoms and almost zero interaction between $\mathrm{H}$ atoms. Thus one can expect different trapping mechanisms for $\mathrm{H}$ and $\mathrm{He}$. Indeed, the qualitative difference can be seen from a visualisation of the atomic configurations presented in figure 5. In case of $\mathrm{H}$ atoms, an aggregation with the local hydrogen concentration up to five times higher as compared to the bulk average was formed promoting the $\mathrm{W}$ atom ejection within the aggregation volume. At the same time, helium atoms were found to cluster in the very beginning of the simulation, thus causing significant lattice distortion and facilitating the tungsten atom ejection.

The results of the second series of MD simulations revealing the critical $\mathrm{H}$ concentration at the moment of the first tungsten atom ejection in the presence of $\mathrm{He}_{\mathrm{M}}$ clusters in the system are shown in figure 6. For every considered combination of the initial number of He atoms and HIR the number of successful simulation runs varied from eight to 10 with the exception of simulations with six He atoms with low and middle values of HIR, where the number of successful runs was two. As in the case of pure $\mathrm{H}$ simulations and due to the small number of data points, the box-and-whiskers plot [38] was used to represent the data. 
(a)

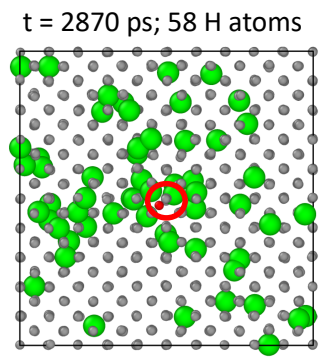

- bulk tungsten (b)

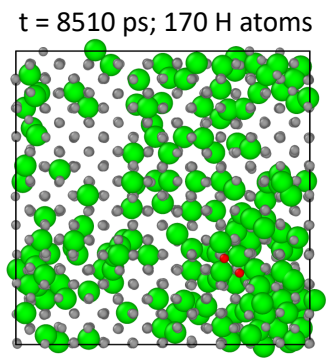

initial vacancy (c)

$\mathrm{t}=2860 \mathrm{ps} ; 57 \mathrm{H}$ atoms

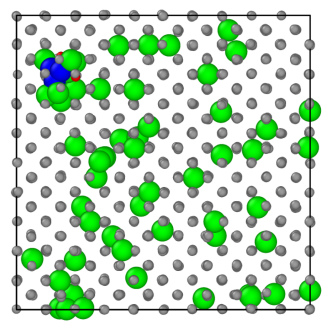

hydrogen helium

Figure 5: Simulation box snapshots taken at the moment of the first tungsten atom ejection (bubble nucleation) by $\mathrm{H}$ atoms, HIR $=10 \mathrm{appm} / \mathrm{ps}$. Grey atoms - bulk tungsten, red atoms - tungsten SIA, green atoms - hydrogen, blue atoms - helium. Simulation results with three different starting configurations are shown. The time when each snapshot was taken and the corresponding number of $\mathrm{H}$ atoms in the simulation box is written on the top of each image. (a) The crystal with an initial vacancy (shown with red circle) and no He atoms. The first tungsten atom is ejected at comparatively low concentration of $\mathrm{H}$ (see figure 4. (b) A defect-free lattice and no He atoms present. A cloud of $\mathrm{H}$ atoms has been formed around the vacant lattice site, the critical average concentration is higher than in (a) (see figure 6). (c) A defect-free crystal and three initially present He atoms which have already formed a cluster prior to the $\mathrm{W}$ atom ejection. 
For the two cases where the number of data points in the dataset was two, the raw data was plotted (coloured stars) since the box-and-whiskers plot for such datasets is misleading. For better visualisation of the trend of critical $\mathrm{H}$ concentration evolution, a red dashed line connecting median values for HIR = $10 \mathrm{appm} / \mathrm{ps}$ was added.

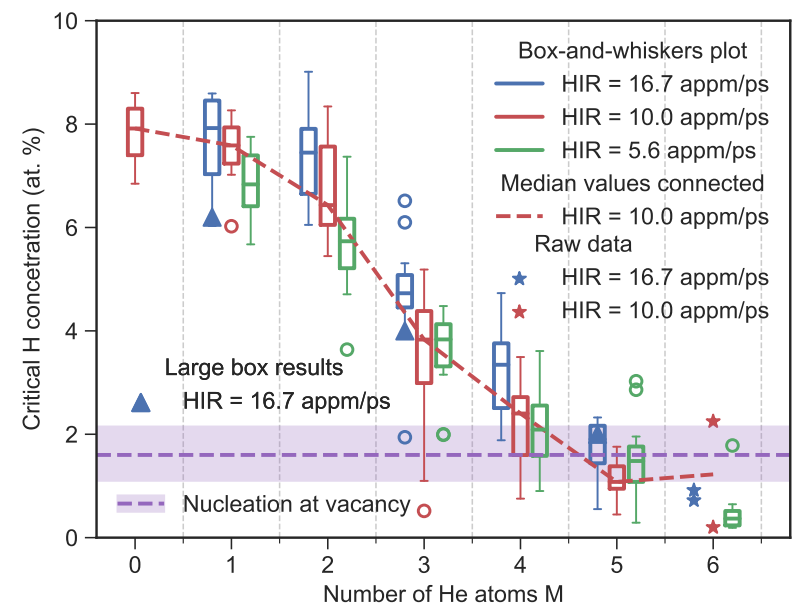

Figure 6: The critical average atomic $\mathrm{H}$ concentration variation with the number of He atoms in the $\mathrm{W}$ crystal for three values of HIR represented with three coloured box-and-whiskers plots as it was done in the figure 4 The coloured circles represent the outliers and coloured stars represent the raw data for the cases where using the box-and-whiskers plot would be misleading due to the low number of points in the dataset. The median values for HIR = $10 \mathrm{appm} / \mathrm{ps}$ are connected with the red dashed line. Purple horizontal dashed line and range represent the median value and IQR for the simulations without He atoms and with preexisting vacancy for $\mathrm{HIR}=10 \mathrm{appm} / \mathrm{ps} \mathrm{ps} /$ atom (see figure 4). Blue triangles show the data obtained using larger simulation box of size $20 \times 20 \times 20 a_{0}^{3}$ with HIR $=16.7 \mathrm{ps} /$ atom.

The results presented in figure 6 show a decrease of the value of critical $\mathrm{H}$ concentration with an increase of the number of helium atoms present in the simulation box. When the number of He atoms in the $\mathrm{He}_{\mathrm{M}}$ cluster exceed four, the critical $\mathrm{H}$ concentration becomes similar to the case of pre-existing vacancy shown by purple horizontal line (see figure 4). The spread of the data allows 
the factor of eight, thus showing the clear evidence of the synergy between hydrogen and helium atoms when they eject a tungsten atom. At the same time the hydrogen insertion interval presented by different colours in the figure has a small effect as it was in case of simulations without $\mathrm{He}_{\mathrm{M}}$ clusters and with pre-existing vacancy (see figure 4 .

The helium atoms were found to bond together at the very beginning of the simulation, thus causing lattice distortion and facilitating the tungsten atom ejection. It was concluded that if more than four He atoms were present in the computation cell, then the tungsten atom ejection was not primarily driven by of small clusters of $\mathrm{He}$ atoms in the material during $\mathrm{H}$ implantation enhances the $\mathrm{H}$ bubble formation. Clusters containing more than four He atoms act as additional traps for $\mathrm{H}$ atoms and can be as strong as vacancies.

A limited number of simulations was done in a larger box size of $20 \times 20 \times 20 a_{0}^{3}$ with HIR $=16.7 \mathrm{ps} /$ atom in order to estimate the effect of the size of the system on the value of critical hydrogen concentration. The obtained results for 1,3 and $5 \mathrm{He}$ atoms in the system are shown on the figure 6 with blue triangles. As can be seen from figure 6, the moment of the first tungsten atom ejection (in terms of the average atomic concentration of hydrogen in the crystal) matches well with the data obtained with the smaller box. Thus it was concluded that the variation of the simulations system size does not affect the obtained results significantly.

So far the main focus of this work was the moment of the first Frenkel pair formation. However it is also important to investigate the subsequent dynamics of bubble formation. The number of detected vacancies as a function of time (bottom $\mathrm{X}$ axis) and $\mathrm{H}$ concentration (top $\mathrm{X}$ axis) for the case of HIR of 10 appm/ps (corresponding flux $=2.03 \times 10^{27} \frac{1}{m^{2} s}$ ) is shown in figure 7 . The colour of the solid lines shows the number of He atoms $\mathrm{M}$ present in the $\mathrm{He}_{\mathrm{M}}$ cluster in the system. The dashed vertical lines show the median values for the 
is plotted with red dashed line in figure6.6.

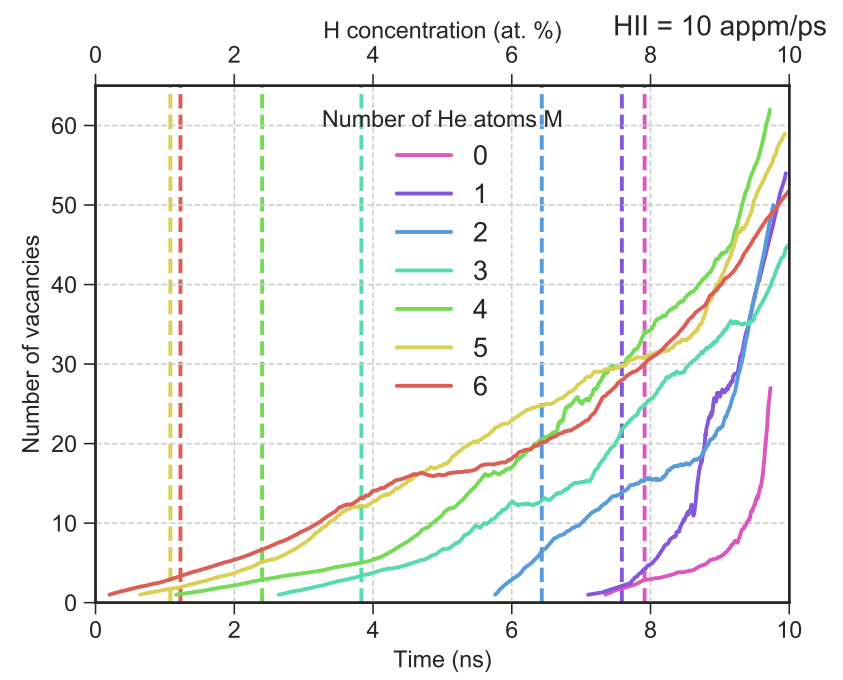

Figure 7: The evolution of the amount of vacancies with time for different number of He atoms present in the simulation. The solid lines represent the vacancy concentration with the colour corresponding to a certain number of He atoms in the cluster. The bottom $\mathrm{X}$ axis shows time and the top $\mathrm{X}$ axis shows the corresponding $\mathrm{H}$ concentration in the system. The results only for HIR $=10 \mathrm{appm} / \mathrm{ps}$ (corresponding flux $=2.03 \times 10^{27} \frac{1}{m^{2} s}$ ) are shown. Dashed vertical lines represent the median values of critical $\mathrm{H}$ concentration for the first Frenkel pair formation and show the same data as the red dashed line in Figure6

As can be seen from figure 7, for the simulations with number of He atoms lower than three there is a rapid increase in the rate of the vacancy formation (slope of the curve) at the very end of the simulation when the value of the $\mathrm{H}$ concentration reaches nine percent. At the same time this effect is less pronounced in the simulations with higher number of He atoms in the system showing a steady increase of the amount of vacancies in the system during the simulations. Moreover, in the second half of the simulated time, when the $\mathrm{H}$ concentration is higher than five percent the curves for six, five and four He atoms show practically identical trends. Thus the presence of $\mathrm{He}_{\mathrm{M}}$ clusters in the simulation affects not only the initial Frenkel pair formation mechanism but also the subsequent bubble growth. However, in order to reach experimental 
time and length scales and give quantitative estimations of the possible effects such as whether He created defects act as additional traps increasing $\mathrm{H}$ retention or form a subsurface diffusion barrier for $\mathrm{H}$ and thus reducing the overall $\mathrm{H}$ retention, one has to perform simplified mean field theory simulations of mixed He-H implantation in the material. Together with our previous study reporting diffusion parameters of $\mathrm{He}_{\mathrm{M}}$ and mixed $\mathrm{He}_{\mathrm{M}}-\mathrm{H}_{\mathrm{N}}$ clusters in $\mathrm{W}$ [27, $\mathrm{MS}$ and MD simulations presented in this paper provide an insight about the $\mathrm{H}$ assisted Frenkel pair formation mechanism in the presence of $\mathrm{He}_{\mathrm{M}}$ clusters and provide the necessary parametrisation for upper scale simulations.

\section{Conclusions}

A set of MS and MD simulations was performed in order to investigate $\mathrm{He}$ and $\mathrm{H}$ trapping properties under mixed $\mathrm{He}-\mathrm{H}$ plasma implantation. Two versions of an EAM potential for the W-H-He system by Bonny et al. 30 in the framework of an EAM model and a BOP potential by Li et al. [28 29] were validated by comparison of the static calculations with ab initio data from the literature. Based on the results of the static simulations the following conclusions can be drawn.

325

Results for the stability of $\mathrm{He}_{\mathrm{M}}$ clusters revealed that the EAM1 potential shows rapid increase of He binding energy with increase of the number of $\mathrm{He}$ atoms $\mathrm{M}$ in the cluster $\mathrm{He}_{\mathrm{M}}$ and significantly overestimates the value of the binding energy as compared to ab initio results. At the same time EAM2 and BOP potentials show good agreement.

330 Results of the stability of $\mathrm{He}_{\mathrm{M}}-\mathrm{H}$-Vac clusters showed that the BOP potential overestimates the binding energy almost by a factor of two for clusters containing one to three He atoms, while both EAM potentials give reasonable agreement.

Results for the $\mathrm{He}_{\mathrm{M}}$ cluster assisted Frenkel pair formation demonstrated that all the considered potentials provide similar values for the formation energy in good agreement with $a b$ initio data. However due to the poor performance of the $\mathrm{BOP}$ potential regarding the stability of $\mathrm{He}_{\mathrm{M}}$ clusters and unsatisfac- 
tory results of EAM1 regarding the stability of $\mathrm{He}_{\mathrm{M}}-\mathrm{H}-\mathrm{Vac}$ clusters, the EAM2 potential was chosen for finite temperature MD simulations.

The effect of $\mathrm{He}_{\mathrm{M}}$ cluster size and the $\mathrm{H}$ implantation regime (HIR value) 340 on the $\mathrm{H}$ bubble formation mechanism in bulk $\mathrm{W}$ was investigated by means of MD simulations using the EAM2 potential. As a result of the simulations it is possible to conclude the following.

No significant effect on $\mathrm{H}$ bubble nucleation mechanism in tungsten was found when $\mathrm{H}$ insertion rate was varied from 5.6 to $50 \mathrm{appm} / \mathrm{ps}$ irrespective of the presence of $\mathrm{He}_{\mathrm{M}}$ clusters. He atoms clustered in the very beginning of the simulations and stayed clustered during the rest of the simulation due to the high value of the binding energy. The presence of $\mathrm{He}_{\mathrm{M}}$ clusters in the material facilitates defect formation by decreasing the critical $\mathrm{H}$ concentration needed for Frenkel pair formation and also affects the subsequent bubble growth mechanism. This is explained by the lattice strain induced by the $\mathrm{He}_{\mathrm{M}}$ clusters, which ease the ejection of $\mathrm{W}$ atom by addition of several $\mathrm{H}$ atoms to the $\mathrm{He}_{\mathrm{M}}$ cluster.

The performed simulations suggest that the presence of $\mathrm{He}_{\mathrm{M}}$ clusters in the material during mixed $\mathrm{He}-\mathrm{H}$ plasma exposure facilitates $\mathrm{H}$ trapping and bubble formation and growth. On top of that, due to the self-trapping mechanism $\mathrm{He}_{\mathrm{M}}$ clusters can create vacancies thus being the additional source of traps for $\mathrm{H}$. The results reported in this paper provide a thorough insight into the mechanisms that determine $\mathrm{H}$ retention in $\mathrm{W}$ in the presence of $\mathrm{He}$ in the material that can be used to interpret the results of experiments with both simultaneous 39] and subsequent [40 $\mathrm{H}$ and He plasma exposures. However, in order to reach experimental time and length scales and investigate the effect of all the considered mechanisms on the $\mathrm{H}$ retention one has to use simplified methods with reduced number of degrees of freedom such as mean field and coarse grained approximations 41 43] or Kinetic Monte Carlo simulations 44. Together with the data for diffusion and thermal stability of mixed $\mathrm{He}_{\mathrm{M}}-\mathrm{H}_{\mathrm{N}}$ clusters reporter earlier [27, this paper provides the parameters needed for such simulations. 


\section{Acknowledgements}

This work was supported by the European Commission and carried out within the framework of the Erasmus Mundus International Doctoral College in Fusion Science and Engineering (FUSION-DC).

\section{References}

[1] R. E. Clark, D. Reiter, Nuclear fusion research: understanding plasmasurface interactions, volume 78, Springer, 2005.

[2] R. Joachim, T. Emmanuelle, L. Thierry, P. Volker, B. Sebastijan, L. Alberto, F. C. Glenn, P. D. Russell, S. Klaus, V. O. Olga, A. C. Rion, Tritium inventory in iter plasma-facing materials and tritium removal procedures, Plasma Physics and Controlled Fusion 50 (2008) 103001. URL: http://stacks.iop.org/0741-3335/50/i=10/a=103001.

[3] H. Y. Xu, G. N. Luo, H. Schut, Y. Yuan, B. Q. Fu, A. Godfrey, W. Liu, G. D. Temmerman, Enhanced modification of tungsten surface by nanostructure formation during high flux deuterium plasma exposure, Journal of Nuclear Materials 447 (2014) 22-27. URL: http: //www.sciencedirect.com/science/article/pii/S0022311513012968 doi http://dx.doi.org/10.1016/j.jnucmat.2013.12.010.

[4] J. P. Sharpe, R. D. Kolasinski, M. Shimada, P. Calderoni, R. A. Causey, Retention behavior in tungsten and molybdenum exposed to high fluences of deuterium ions in TPE, Journal of Nuclear Materials 390391 (2009) 709-712. URL: http://www.sciencedirect.com/science/ a article/pii/S0022311509002153 doi/http://dx.doi.org/10.1016/j. jnucmat.2009.01.195

[5] W. M. Shu, G. N. Luo, T. Yamanishi, Mechanisms of retention and blistering in near-surface region of tungsten exposed to high flux deuterium plasmas of tens of eV, Journal of Nuclear Materials 367-370, Part 
395 प

I

$$
\text { jnucmat.2004.10.137. }
$$

[8] L. Buzi, Influence of the Particle Flux on Surface Modifications of Tungsten,

[9] B. Tyburska, Deuterium Retention in Carbon and Self-Implanted Tungsten, Report, Max-Planck-Institut für Plasmaphysik, 2010.

[10] Y. Zayachuk, M. H. J. . t. Hoen, P. A. Z. v. Emmichoven, D. Terentyev, I. Uytdenhouwen, G. v. Oost, Surface modification of tungsten and tungsten-tantalum alloys exposed to high-flux deuterium plasma and its impact on deuterium retention, Nuclear Fusion 53 (2013) 013013. URL: http://stacks . iop.org/0029-5515/53/i=1/a=013013.

[11] Y. Zayachuk, Deuterium Retention in Tungsten and Tungsten-Tantalum Alloys under High Flux Plasma Exposure ('Deuteriumretentie in wolfraam en wolfraam-tantaallegeringen onder blootstelling aan een hoge plasmaflux'), Thesis, Ghent University, 2013. 
[12] M. Baldwin, R. Doerner, Helium induced nanoscopic morphology on tungsten under fusion relevant plasma conditions, Nuclear Fusion 48 (2008) 035001. URL: http://stacks.iop.org/0029-5515/48/i=3/ 425 a a=035001?key=crossref . ab90d98340142ce1fd8761231da9d039. doi 10 . 1088/0029-5515/48/3/035001.

[13] M. J. Baldwin, R. P. Doerner, Formation of helium induced nanostructure 'fuzz' on various tungsten grades, Journal of Nuclear Materials 404 (2010) 165-173. doi $10.1016 / j \cdot j$ jucmat.2010.06.034.

${ }_{430}$ [14] M. Yamagiwa, S. Kajita, N. Ohno, M. Takagi, N. Yoshida, R. Yoshihara, W. Sakaguchi, H. Kurishita, Helium bubble formation on tungsten in dependence of fabrication method, in: Journal of Nuclear Materials, volume 417, 2011, pp. 499-503. doi 10.1016/j.jnucmat.2011.02.007.

[15] Y. Ueda, M. Fukumoto, J. Yoshida, Y. Ohtsuka, R. Akiyoshi, H. Iwakiri, N. Yoshida, Simultaneous irradiation effects of hydrogen and helium ions on tungsten, Journal of Nuclear Materials 386-

a 388 (2009) 725-728. URL: http://www.sciencedirect.com/science/

a article/pii/S0022311508009690. doi/http://dx.doi.org/10.1016/j. jnucmat.2008.12.300.

${ }_{440}$ [16] M. Miyamoto, D. Nishijima, M. J. Baldwin, R. P. Doerner, Y. Ueda, K. Yasunaga, N. Yoshida, K. Ono, Microscopic damage of tungsten exposed to deuterium-helium mixture plasma in PISCES and its impacts on retention property, Journal of Nuclear Materials 415 (2011)

a S657-S660. URL: http://www.sciencedirect.com/science/article/

445 pii/S0022311511000201. doi http://dx.doi.org/10.1016/j.jnucmat. 2011.01 .008

[17] G.-H. Lu, H.-B. Zhou, C. S. Becquart, A review of modelling and simulation of hydrogen behaviour in tungsten at different scales, Nuclear Fusion 54 (2014) 086001. URL: http://stacks. iop.org/0029-5515/54/i=8/a= 450 086001 
[18] J. Marian, C. S. Becquart, C. Domain, S. L. Dudarev, M. R. Gilbert, R. J. Kurtz, D. R. Mason, K. Nordlund, A. E. Sand, L. L. Snead, T. Suzudo, B. D. Wirth, Recent advances in modeling and simulation of the exposure and response of tungsten to fusion energy conditions, Nuclear Fusion 57 (2017) 092008. URL: http://stacks.iop.org/0029-5515/57/i=9/a= 092008 .

[19] B. D. Wirth, K. Hammond, S. Krasheninnikov, D. Maroudas, Challenges and opportunities of modeling plasmasurface interactions in tungsten using high-performance computing, Journal of $\mathrm{Nu}-$ clear Materials 463 (2015) 30-38. URL: http://www.sciencedirect. 1. com/science/article/pii/S0022311514008757, doi/https://doi.org/ $10.1016 / \mathrm{j} \cdot \mathrm{jnucmat} .2014 .11 .072$.

[20] Y.-L. Liu, Y. Zhang, G. N. Luo, G.-H. Lu, Structure, stability and diffusion of hydrogen in tungsten: A first-principles study, Jour465 nal of Nuclear Materials 390-391 (2009) 1032-1034. URL: http: //www.sciencedirect.com/science/article/pii/S0022311509002918 doi http://dx.doi.org/10.1016/j.jnucmat.2009.01.277.

[21] K. Heinola, T. Ahlgren, K. Nordlund, J. Keinonen, Hydrogen interaction with point defects in tungsten, Physical Review B: Condensed Matter 82 (2010) 094102. URL: http://link.aps.org/doi/10.1103/PhysRevB.82. 094102

[22] D. F. Johnson, E. A. Carter, Hydrogen in tungsten: Absorption, diffusion, vacancy trapping, and decohesion, Journal of Materials Research 25 (2010) 315-327. URL: http://dx.doi.org/10.1557/JMR.2010.0036. 475 doi doi:10.1557/JMR.2010.0036.

[23] C. S. Becquart, C. Domain, Migration energy of He in W revisited by ab-initio calculations, Physical Review Letters 97 (2006) 196402. URL: http://link.aps.org/doi/10.1103/PhysRevLett.97.196402. 
[24] C. S. Becquart, C. Domain, A density functional theory assessment of the clustering behaviour of $\mathrm{He}$ and $\mathrm{H}$ in tungsten, Journal of Nuclear Materials 386-388 (2009) 109-111. URL: http: //www.sciencedirect.com/science/article/pii/S0022311508008179 doi:http://dx.doi.org/10.1016/j.jnucmat.2008.12.085

[25] H.-B. Zhou, Y.-L. Liu, S. Jin, Y. Zhang, G.-N. Luo, G.-H. Lu, Towards suppressing $\mathrm{H}$ blistering by investigating the physical origin of the $\mathrm{H}-\mathrm{He}$

口 interaction in W, Nuclear Fusion 50 (2010) 115010. URL: http://stacks. iop.org $/ 0029-5515 / 50 / i=11 / a=115010$.

[26] A. Takayama, A. M. Ito, Y. Oda, H. Nakamura, First principles investigation of cluster consisting of hydrogen-helium atoms interstitially-trapped in tungsten, Journal of Nuclear Materials

1 463 (2015) 355-358. URL: http://www.sciencedirect.com/science/

a article/pii/S002231151400782X. doi/http://dx.doi.org/10.1016/j. jnucmat.2014.11.001.

[27] P. Grigorev, D. Terentyev, G. Bonny, E. E. Zhurkin, G. van Oost, J.-M. Noterdaeme, Mobility of hydrogen-helium clusters in tungsten studied by molecular dynamics, Journal of Nuclear Materials 474 (2016) 143-149. URL: http://linkinghub.elsevier.com/retrieve/ pii/S0022311516300952, doi:10.1016/j.jnucmat.2016.03.022.

[28] X.-C. Li, X. Shu, Y.-N. Liu, F. Gao, G.-H. Lu, Modified analytical interatomic potential for a $\mathrm{W}-\mathrm{H}$ system with defects, Journal of Nuclear Mate-

a rials 408 (2011) 12-17. URL: http://www.sciencedirect.com/science/

a article/pii/S0022311510006008 doi/http://dx.doi.org/10.1016/j. jnucmat.2010.10.020.

[29] X.-C. Li, X. Shu, Y.-N. Liu, Y. Yu, F. Gao, G.-H. Lu, Analytical $\mathrm{W}-\mathrm{He}$ and $\mathrm{H}-\mathrm{He}$ interatomic potentials for a $\mathrm{W}-\mathrm{H}-\mathrm{He}$ sysn tem, Journal of Nuclear Materials 426 (2012) 31-37. URL: http: 
//www.sciencedirect.com/science/article/pii/S0022311512001602 doi http://dx.doi.org/10.1016/j.jnucmat.2012.03.039.

[30] G. Bonny, P. Grigorev, D. Terentyev, On the binding of nanometric

[31] M. C. Marinica, V. Lisa, M. R. Gilbert, L. Proville, S. L. Dudarev, J. Marian, G. Bencteux, F. Willaime, Interatomic potentials for modelling radi-

[32] G. Bonny, D. Terentyev, A. Bakaev, P. Grigorev, D. V. Neck, Many-body central force potentials for tungsten, Modelling and Simulation in Materiorg $/ 0965-0393 / 22 / i=5 / a=053001$.

[33] S. Plimpton, Fast parallel algorithms for short-range molecular dynamics, Journal of Computational Physics 117 (1995)

[34] J. P. Gunn, S. Carpentier-Chouchana, F. Escourbiac, T. Hirai, S. Panayotis, R. A. Pitts, Y. Corre, R. Dejarnac, M. Firdaouss, M. Kočan, M. Komm, A. Kukushkin, P. Languille, M. Missirlian, W. Zhao, G. Zhong, Surface heat loads on the ITER divertor vertical targets, Nuclear Fusion 57 (2017) 46025. URL: http://stacks.iop.org/0029-5515/57/i=4/a= 046025,

[35] F. Sefta, K. D. Hammond, N. Juslin, B. D. Wirth, Tungsten surface evolution by helium bubble nucleation, growth and rupture, Nuclear Fusion I 53 (2013) 073015. URL: http://stacks.iop.org/0029-5515/53/i=7/a= 535 073015 
[36] P. Grigorev, D. Terentyev, G. Bonny, E. E. Zhurkin, G. Van Oost, J. M. Noterdaeme, Interaction of hydrogen with dislocations in tungsten: An atom-

1]

540

[37] J. Boisse, C. Domain, C. S. Becquart, Modelling self trapping and trap mutation in tungsten using DFT and Molecular Dynamics with an empirical potential based on DFT, Journal of Nuclear Materials 455 545

[41] Xolotl plasma-surface interactions, 2018. URL: https://sourceforge. net/projects/xolotl-psi/.

(2014) 10-15. URL: http://www.sciencedirect.com/science/article/ pii/S0022311514001032, doi/http://dx.doi.org/10.1016/j.jnucmat. 2014.02 .031

[38] J. W. Tukey, Exploratory data analysis, Adisson-Wesley, New Tork, 1977.

[39] L. Buzi, G. De Temmerman, A. E. Huisman, S. Bardin, T. W. Morgan, M. Rasinski, R. A. Pitts, G. Van Oost, Response of tungsten surfaces to helium and hydrogen plasma exposure under ITER relevant steady state and repetitive transient conditions, Nuclear Fusion 57 (2017) 126009. URL: http://stacks.iop.org/0029-5515/57/i=12/a=126009 doi $10.1088 / 1741-4326 /$ aa81e4

[40] S. Markelj, T. Schwarz-Selinger, A. Založnik, Hydrogen isotope accumulation in the helium implantation zone in tungsten, Nuclear Fusion 57 (2017) 064002. URL: http://stacks.iop.org/0029-5515/57/i=6/a= 064002, doi $10.1088 / 1741-4326 /$ aa6b27. Oost, J.-M. Noterdaeme, Numerical analysis of TDS spectra under high and low flux plasma exposure conditions, Physica Scripta 2016 (2016) 014039. URL: http://stacks .iop.org/1402-4896/2016/i=T167/a=014039. 
[43] E. Hodille, F. Ghiorghiu, Y. Addab, A. Zalonik, M. Minissale, Z. Piazza, C. Martin, T. Angot, L. Gallais, M.-F. Barthe, C. Becquart, S. Markelj, J. Mougenot, C. Grisolia, R. Bisson, Retention and release of hydrogen isotopes in tungsten plasma-facing components: the role of grain boundaries and the native oxide layer from a joint experiment-simulation integrated approach, Nuclear Fusion 57 (2017) 076019. URL: http://stacks.iop. org $/ 0029-5515 / 57 / i=7 / a=076019$

[44] C. Domain, C. Becquart, L. Malerba, Simulation of radiation damage in Fe alloys: an object kinetic Monte Carlo approach, Journal of Nu-

n clear Materials 335 (2004) 121-145. URL: http://www.sciencedirect.

a com/science/article/pii/S0022311504006385. doi/https://doi.org/ $10.1016 / \mathrm{j} \cdot \mathrm{jnucmat} .2004 .07 .037$. 